\title{
Methodology for the manufacture of smart composites with thermoplastic matrix
}

\author{
L. Elsoufi ${ }^{1,2}$, K. Khalil ${ }^{3}$, R. Lachat ${ }^{1}$, W. Charon ${ }^{1}$ \& M. Zoaeter ${ }^{2}$ \\ ${ }^{1}$ University of Technology of Belfort-Montbeliard (UTBM), \\ M3M Laboratory, France \\ ${ }^{2}$ Lebanese University (UL), LPM laboratory, Lebanon \\ ${ }^{3}$ Centre universitaire de technologie (CUT), Lebanon
}

\section{Abstract}

The adaptable mechanical structures in the form of Shell have found large developments and use in many applications, especially in the field of smart structures where the piezoelectric components are used as actuators and sensors. Many functional constraints prevent the control of a structure by elements reported on the surfaces of the object. Thus, the piezoelectric components necessary for the control of the structures will be integrated into this, i.e., in material, even the material of the wall. Certain work describes the manner and the performances of structures by using composite materials with thermoset matrices as structural support and piezoelectric components as the actuator or sensor elements. The current difficulties in recycling the thermoset materials are hindering the industrial development of such structures. For this reason, we propose to use composites with a thermoplastic matrix. Unfortunately, the current processes of achieving models in smart thermoplastic structures are not directly exploitable for the integration of components such as the piezoelectric actuators and sensors which are fragile and sensitive to temperature.

This work evaluates the sizes typically reached in the process transformations of such composites with a thermoplastic matrix in order to be able to establish the behavior models for the realized structures. A thermo-mechanic testing method using dynamic mechanical analysis (DMA) is also proposed.

Keywords: smart thermoplastic manufacturing, cooling rate, thermoplastic filling time, PZT sensor, piezoelectric properties degradation. 


\section{Introduction}

Recent applications in smart structures and materials are limited by the manufacture of composites with a thermosetting matrix [2] such as: shape control of airplane wings [3], car bodies, reflector antennas [4], deformable mirrors, and actuators such as the so-called bimorph and C-block actuators [5, 6], which are widely used in the automation and aeronautics industries.

This study is a contribution to modeling in smart thermoplastic structures manufacturing which have a great importance in recycling. The relatively high viscosity of melted thermoplastic materials leads the manufacturers of composites with thermoplastic matrix to raise the temperature of these materials during the manufacturing processes until temperatures can reach 1.5 to 2 times the melting point of these materials [1]. Among the processes we can enumerate: multi-material injection, multiphase injection, rolling, and thermoforming. The integration of components like memory-shape alloys such as the piezoelectric actuators or sensors in composite parts with thermoplastic matrix causes the thermal limit to exceed that of these components $T_{c} / 2\left(T_{c}\right.$ : Curie temperature of the piezoelectric component) which varies according to the type of piezoelectric.

There are recent piezoelectric materials which have relatively high curie temperatures compared to those traditional ones [7], such as: $0.36 \mathrm{BiScO}_{3}$ $0.64 \mathrm{PbTiO}_{3}$ and $0.3 \mathrm{BiYbO}_{3}-0.7 \mathrm{PbTiO}_{3}$. However, these have not been commercialized yet because they are still under research.

The aim of this study is to determine the relationship between the thermoplastic manufacturing conditions and those of commercial traditional piezoelectric materials. In this context, experimental tests have been carried out on piezoceramic plates by the dynamical-mechanical analysis test machine (DMA) which is called "Viscoanalyzer". In this work, we will analyze the curvetype of temperature variation over time in different conditions of fabrication using simulation under ANSYS. The thermoplastic manufacturing process treated in this study is the injection molding of a rectangular model, in which we integrate a piezoelectric sensor. We vary the model thickness and the sensor position in order to study their thermal influence on the sensor and to minimize its exposure to heat. This introduction is followed by three sections which represent respectively: Modeling procedures, experimental results, and a conclusion.

\section{Modeling procedures}

In the injection process the thermal phenomena are often the slowest and they mainly control the time cycle as well as the degree of the damage of the piezoelectric component during the process. In practice, the cooling time of a molded part corresponds to the longest phase of the molding cycle as shown in figure 1. The thermal resistance of the piezoelectric sensor requires the knowledge of the thermal phenomena during the molding cycle. In this paper we present the various aspects of the thermal transfers during the thermoplastics injection process. We treat the cooling of the model and the heating of the 
piezoelectric sensor. Furthermore, we introduce concepts of compromise between the heating and cooling of the polymer, and the on-heating of the piezoelectric sensor during the melt plastic filling in the mould. The thermal analysis of the injection process requires the knowledge of three modes of heat transfers: conduction, convection and radiation. The conductive transfers take place in the materials (part and mould). The heat transfer between the wall of the cooling channels and the fluid of the regulation system is controlled by convective transfers.

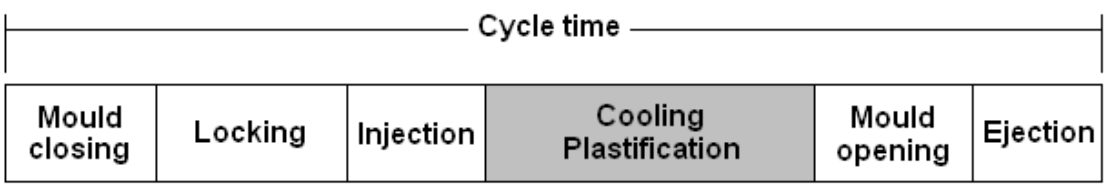

Figure 1: $\quad$ Cycle time.

\subsection{Model description and properties}

The studied model is composed of a thin polypropylene plate (Borealis BD500P) which contains a piezoceramic sensor PZT made of two circular plates: yellow brass and ceramics as shown in figure 2. The polypropylene plate is molded in a steel mould of type AISI 1008. All properties are given by [9].

The properties of polypropylene (Borealis BD500P) are as follows:

- density: $\rho_{2}=800 \mathrm{~kg} / \mathrm{m}^{3}$

- thermal conductivity: $\mathrm{k}_{2}=0.179 \mathrm{~W} / \mathrm{m} . \mathrm{K}$

- specific heat: $\mathrm{c}_{2}=2800 \mathrm{~J} / \mathrm{kg} . \mathrm{K}$

- consistence (degree of viscosity): $\mathrm{K}=11000$

- index of pseudoplasticity: $\mathrm{n}=0.308$

The properties of the steel mould (AISI 1008) are as follows:

- density: $\rho_{1}=7800 \mathrm{~kg} / \mathrm{m}^{3}$

- the thermal conductivity $\left(\mathrm{k}_{1}\right)$ of this type of steel is variable with temperature as shown in the table below:

Table 1: $\quad$ AISI 1008 thermal conductivity.

\begin{tabular}{|l|c|c|c|c|}
\hline Temperature $\left[{ }^{\circ} \mathrm{C}\right]$ & 0 & 100 & 200 & 400 \\
\hline Conductivity $[\mathrm{W} / \mathrm{m} . \mathrm{K}]$ & 65.2 & 60.2 & 54.7 & 45.2 \\
\hline
\end{tabular}

- the specific heat $\left(\mathrm{c}_{1}\right)$ is also variable with temperature as shown in the table below:

Table 2: $\quad$ AISI 1008 specific heat.

\begin{tabular}{|l|c|c|c|c|c|}
\hline Temperature $\left[{ }^{\circ} \mathrm{C}\right]$ & $50-100$ & $150-200$ & $200-250$ & $250-300$ & $350-400$ \\
\hline Specific heat $[\mathrm{J} / \mathrm{kg} . \mathrm{K}]$ & 481 & 519 & 536 & 553 & 595 \\
\hline
\end{tabular}


The properties of the piezoelectric ceramic (PZT upper plate) are as follows:

- density: $\rho_{3}=7500 \mathrm{~kg} / \mathrm{m}^{3}$

- thermal conductivity: $\mathrm{k}_{3}=6 \mathrm{~W} / \mathrm{m} . \mathrm{K}$

- specific heat: $\mathrm{c}_{3}=670 \mathrm{~J} / \mathrm{kg} . \mathrm{K}$

The properties of the yellow brass (PZT lower plate) are as follows:

- density: $\rho_{4}=8750 \mathrm{~kg} / \mathrm{m}^{3}$

- thermal conductivity: $\mathrm{k}_{4}=159 \mathrm{~W} / \mathrm{m} . \mathrm{K}$

- specific heat: $\mathrm{c}_{4}=380 \mathrm{~J} / \mathrm{kg} . \mathrm{K}$

The necessary data to calculation are as follows:

- the mould is controlled using a coolant fluid at the temperature of $\mathrm{T}_{\mathrm{m}}=40^{\circ} \mathrm{C}$

- the injection temperature of polypropylene part is $\mathrm{T}_{\mathrm{M}}=240^{\circ} \mathrm{C}$

- the initial temperature of the piezoelectric sensor is $\mathrm{T}_{\mathrm{p}}=25^{\circ} \mathrm{C}$

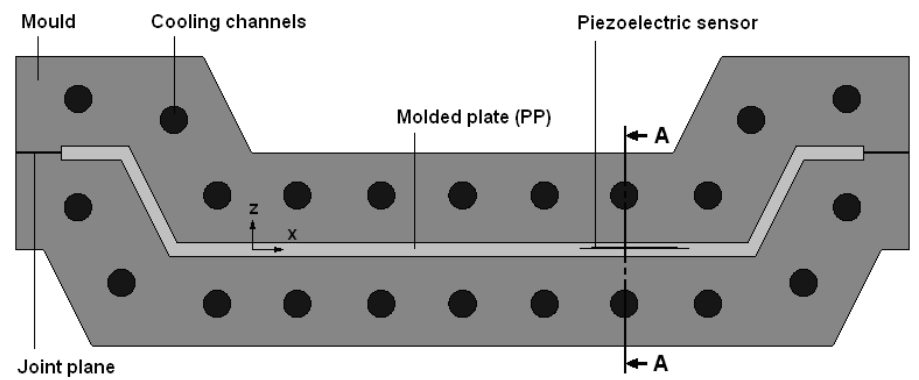

(a) Schematized mould

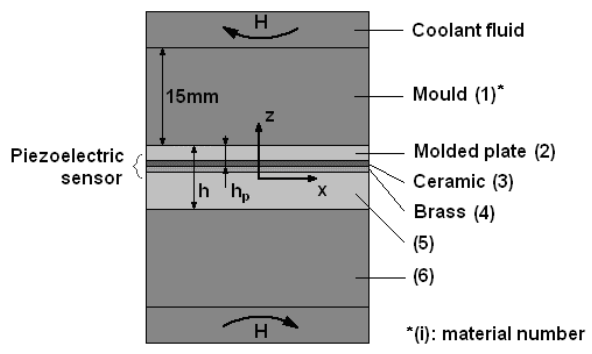

(b) Section A-A : ANSYS model

Figure 2: Description of the mould for simulation.

\subsection{Modeling results}

The modeling of the transient problem is built under ANSYS by studying the thermal distribution over time of the upper part of the molding plate (above the piezoceramic sensor: between sensor and mould) and the piezoceramic sensor during manufacturing at all point in $z$ direction. 
In order to study the several states of manufacturing influence of such composites with thermoplastic matrix on the integrated piezoelectric components, we analyze three sizes for the plate thickness and five positions for the sensor as follows:

$-h=2 \mathrm{~mm}$ with $h_{1}=0.5 \mathrm{~mm}, 0.75 \mathrm{~mm}$ and $1 \mathrm{~mm}$

$-h=5 \mathrm{~mm}$ and $10 \mathrm{~mm}$ with $h_{l}=0.5 \mathrm{~mm}, 0.75 \mathrm{~mm}, 1 \mathrm{~mm}, 1.75 \mathrm{~mm}$ and $2.5 \mathrm{~mm}$

\subsubsection{Part thickness influence}

To illustrate the part thickness influence on the injection thermal cycle and the piezoceramic sensor thermal resistance over time, three simulations were realized for three different part thicknesses $h=2 \mathrm{~mm}, 5 \mathrm{~mm}$ and $10 \mathrm{~mm}$, to the same piezoceramic sensor position $h_{p}=1 \mathrm{~mm}$ as shown in figures 3 and 4 .

In the PP zone between mould and sensor, we notice an important increase in the temperature discard between centre and part surfaces with the increase of the global model thickness. In addition, this model thickness increasing causes an increment in the piezoceramic sensor temperature from $100^{\circ} \mathrm{C}$ to $140^{\circ} \mathrm{C}$.

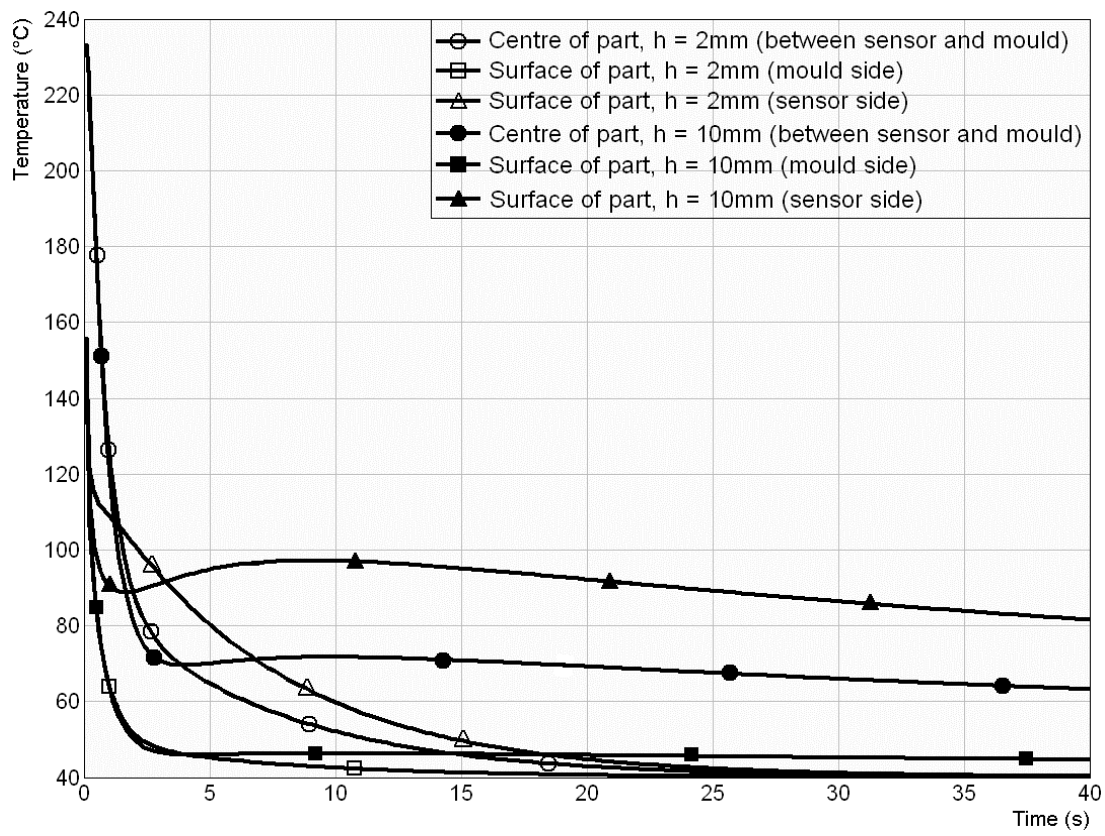

Figure 3: $\quad$ Part thickness influence on plastic zone $\left(h_{p}=1 \mathrm{~mm}\right)$.

\subsubsection{Sensor position influence}

Five simulations were realized on five various piezoceramic sensor positions with aim to study the thermal resistance of the sensor over time under various conditions of manufacture including its positions in model (figures 5 and 6).

We notice a systematic increase in the sensor maximum temperature with its transverse penetration towards the centre of model to reach $160{ }^{\circ} \mathrm{C}$ as shown in figure 6 . 


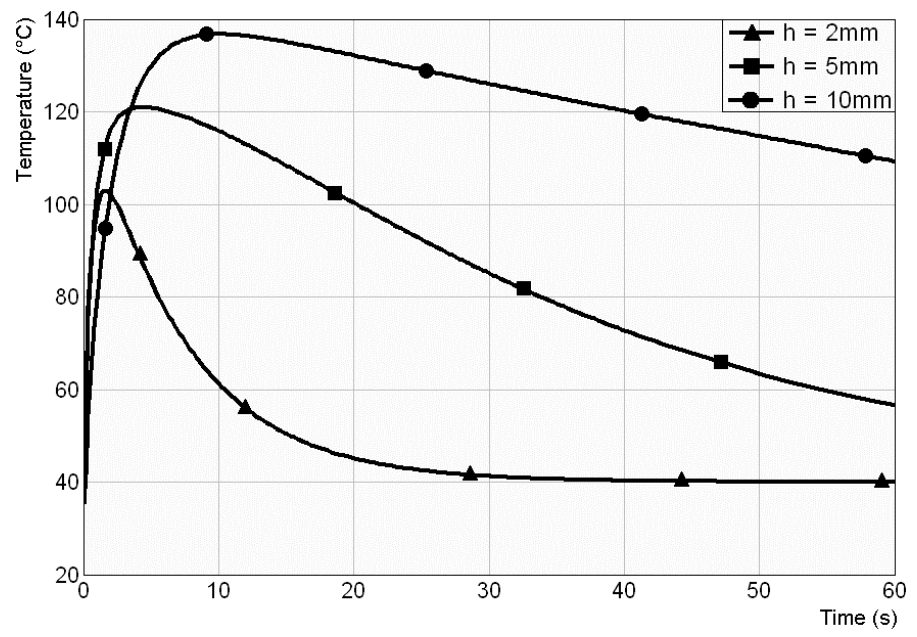

Figure 4: $\quad$ Part thickness influence on piezoceramic sensor $\left(h_{p}=1 \mathrm{~mm}\right)$.

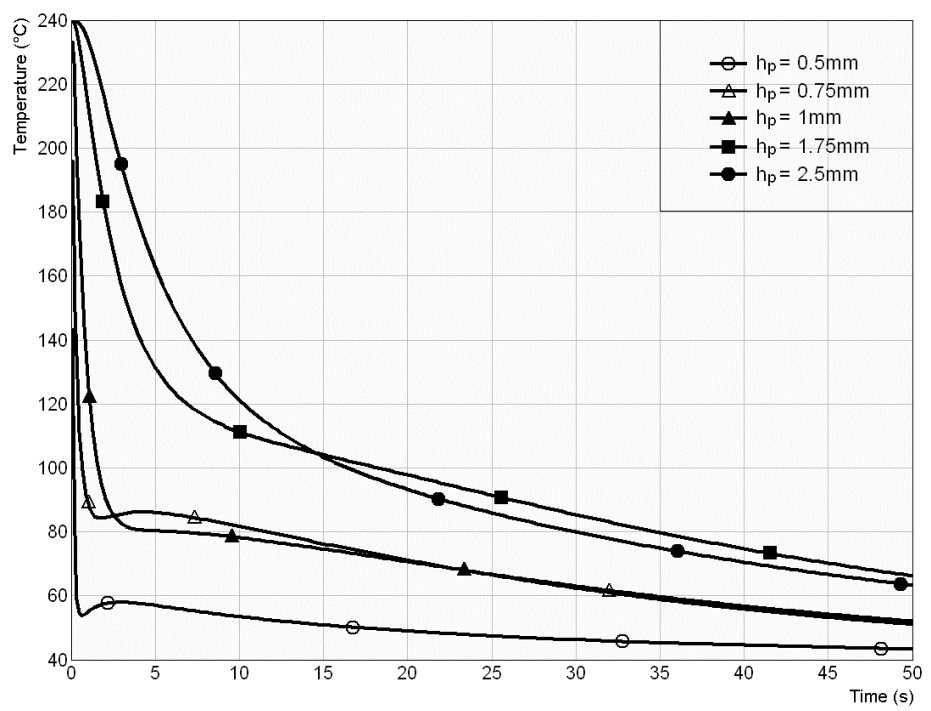

Figure 5: $\quad$ Sensor position influence on plastic zone $(h=5 \mathrm{~mm})$.

\subsubsection{Liquid plastic filling effect}

During the mould filling, a solid layer is formed on the flow surfaces (mould and sensor surfaces), this solidification is able to reduce significantly the available flow passage distance (liquid vein $h_{v}(t)$, fig.7).

The reduction of the liquid vein, related to an increase in the filling rate, causes an important rise in the filling pressure of the mould. However, a significant reduction of the filling time involves an increasing in the pressure due 
to flow increment. Finally, the evolution of the filling pressure related to the filling time, presents successively a decreasing in pressure profile passing by a minimum $P^{*}$ than increasing [8]:

$$
P^{*}\left(z, h_{v}, t^{*}\right)=2 K\left(\frac{2(2 n+1)}{n}\right)^{n}\left(\frac{2 n}{3 n+1}\right)^{-2 n}\left[1-\left(\frac{2 n}{3 n+1}\right)\right]^{-(n+1)} z^{n+1} \frac{h_{p}^{-(3 n+1)}}{\alpha^{-n}}
$$

The optimal filling time values are higher than the ones known in industrial practice. For evident reasons of productivity, it isn't necessary to prolong the filling time until the minimum of the filling pressure. It is preferable to be located at the point that constitutes the best compromise between a significant reduction of the filling time and a minimum increase $\Delta P$ in the filling pressure.

The optimal filling time is the average value of the filling times obtained by decreasing solidified $h_{p}-s(t)$ thickness and increasing solidified thickness $z(t)=\sqrt{\alpha t^{*}}$ as follows [8]:

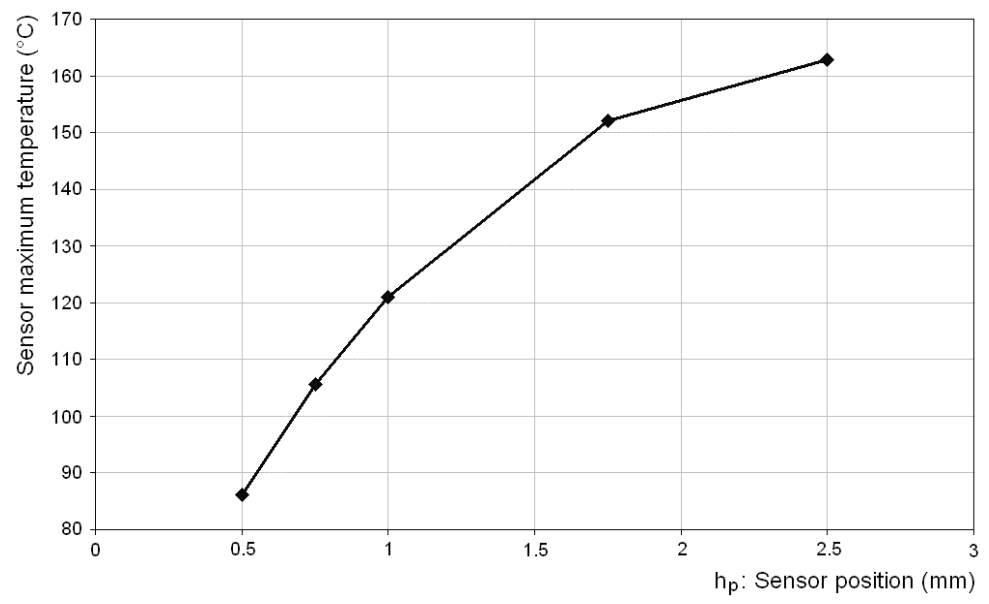

Figure 6: Sensor position influence on piezoceramic sensor $(h=5 \mathrm{~mm})$.

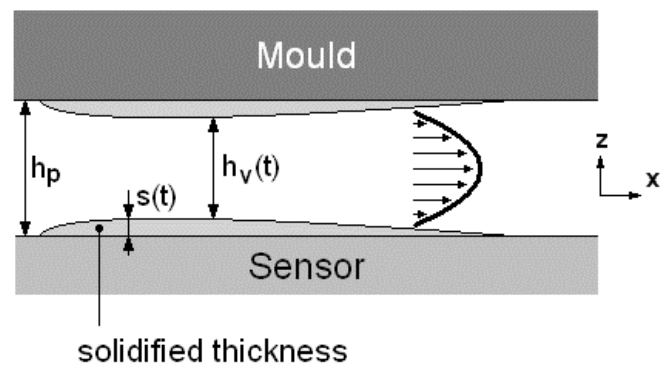

Figure 7: Flow section in the solidification course. 


$$
\begin{aligned}
t_{f}=\left[\frac{\left(\frac{1}{h_{p}}\right)^{\frac{n+1}{n}}+\left(\frac{3 n+1}{h_{p}(n+1)}\right)^{\frac{n+1}{n}}}{2}\right] \times \\
{\left[\frac{\Delta P}{2 K\left(\frac{2(2 n+1)}{n}\right)^{n} x^{n+1}}+\left[\frac{\frac{3 n+1}{h_{p}^{n}}}{\alpha}\left(\frac{2 n}{3 n+1}\right)^{2}\left[1-\left(\frac{2 n}{3 n+1}\right)\right]^{\frac{1}{n}+1}\right]^{-n}\right]^{-\frac{1}{n}} }
\end{aligned}
$$

The filling speed is given by [8] as follows:

$$
\left\{V=\left[\frac{-1.384\left(2 \frac{3 n+1}{n}\right)^{\frac{1}{3}}\left(\frac{a L}{h^{2}}\right)^{\frac{2}{3}}}{\left(\frac{2 n+1}{n}\right)^{n} 2^{n+1} \frac{K \alpha L h^{-n-1}}{k \Delta T}}\right]^{\frac{3}{3 n+2}}\right.
$$

with $\Delta \mathrm{T}=\mathrm{T}_{\mathrm{M}}-\mathrm{T}_{\mathrm{m}}$ and $\mathrm{L}=$ model length.

For a plate with $5 \mathrm{~mm}$ of thickness (PP Borealis BD500P), we obtain $t_{f}=12.6 \mathrm{~s}$ and $V=0.2 \mathrm{~m} / \mathrm{s}$ which allow us to calculate the convection heat transfer coefficient of liquid plastic on the sensor (equal $18 \mathrm{~W} / \mathrm{m}^{2} \mathrm{~K}$ ). This coefficient is necessary to re-modeling the problem in filling conditions.

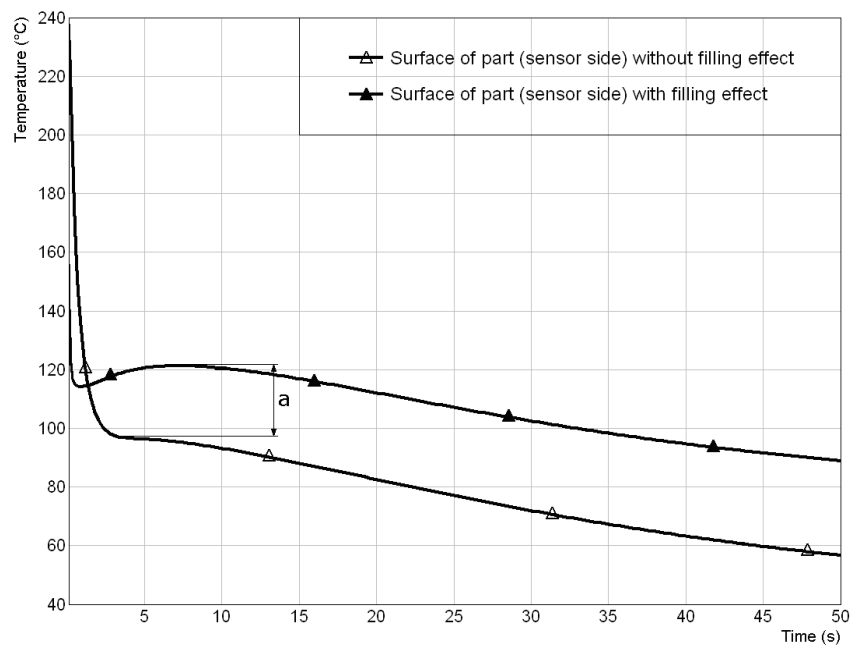

Figure 8: $\quad$ Filling influence on plastic zone $\left(h=5 \mathrm{~mm}, h_{p}=1 \mathrm{~mm}\right)$. 


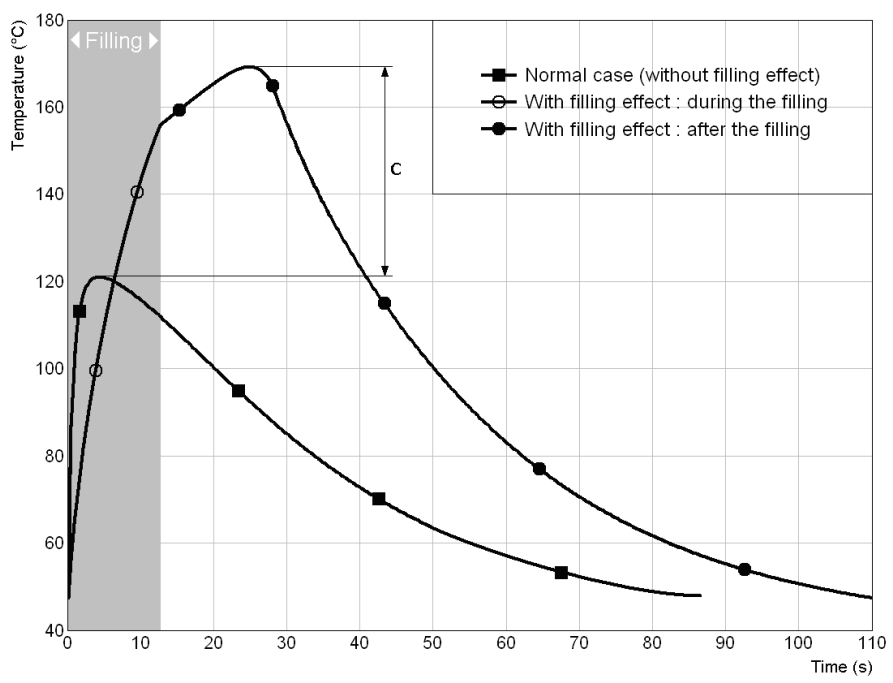

Figure 9: $\quad$ Filling influence on piezoceramic sensor $\left(h=5 \mathrm{~mm}, h_{p}=1 \mathrm{~mm}\right)$.

We notice that the filling process causes a big rise in the temperature maximum values of the different manufacture elements: $20 \%$ of temperature increment (fig.8) in plastic zone (sensor side) and $42 \%$ of sensor temperature increment (fig.9).

The temperature increment and the cooling deceleration play an effective role in the reduction during the creation of solidified thermoplastic thickness on the cold surfaces (mould and sensor). However, the sudden and enormous temperature rise in piezoelectric sensor (figure 9) until exceeding the piezoelectric thermal limit causes the degradation of the sensor i.e. the degradation of these piezoelectric properties (polarization of the piezoelectric ceramic).

\section{Experimental results}

In this work, experimental tests were carried out in M3M laboratory on piezoceramic sensor by a dynamical-mechanical analysis test machine (DMA) which is called "Viscoanalyzer" of type METRAVIB RDS VA 2000, with an acquisition system (TEAC GX-1) used to measure the electric voltage produced by the tested piezoelectric sensor. The viscoanalyzer is a testing machine which makes it possible to determine the viscoelastic properties of materials. The dynamic analysis carried out by this machine allows the thermomechanical performance evaluation of materials and the choice of materials for a function of damping or sound-proofing, for the optimization of the formulation or manufacture processes for the forecast of the products durability. The operation principle consists on applying a sinusoidal force to a sample model and measuring the displacement results. A thermal enclosure makes it possible to cover an experimental window of temperature going from $-150^{\circ} \mathrm{C}$ to $+450^{\circ} \mathrm{C}$. 
The capacity of the machine is about $100 \mathrm{~N}$. The frequency of the test-sample excitation varies from $0.001 \mathrm{~Hz}$ to $200 \mathrm{~Hz}$. The materials tested can be solid, liquid or pasty. Several samples support making it possible to make the following tests: traction, compression, plane or annular shearing, annular pumping, bending, creep or strain relaxation tests.

The principle used in this test installation is based on the relation between the applied sinusoidal displacement and the measured sinusoidal force responding to this displacement (stiffness and loss angle).

The model studied in the experimental test is a circular actuator piezoceramic PZT (same properties in $\$ 2.1$ ), simply supported on the periphery; and applying at the center of the plate a sinusoidal displacement of amplitude $50 \mu \mathrm{m}$ and frequencies: $10,15.85,25.12,39.81,63.1,100 \mathrm{~Hz}$, with temperature variation as the protocol illustrated in figure 10. This experimental protocol allows us to measure the different sensor parameters and the voltage emitted after each temperature crawl up $\left(10^{\circ} \mathrm{C} / \mathrm{min}\right)$ and down $\left(-5^{\circ} \mathrm{C} / \mathrm{min}\right)$ in points $k, l, m$ and $n$ as indicated in figure 10 .

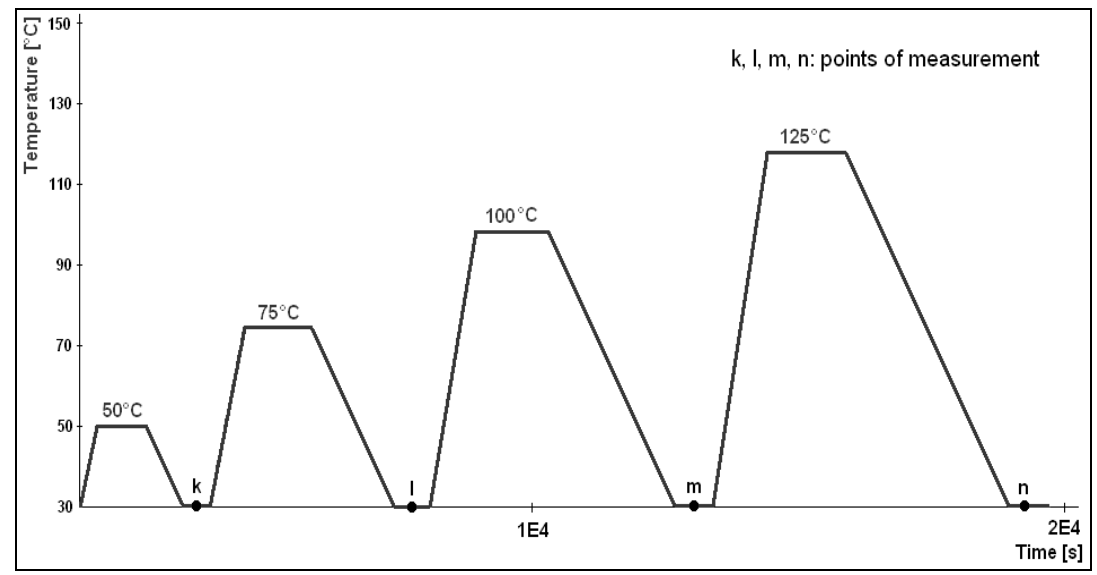

Figure 10: Experimental protocol.

The aim of this experience is to determine the degree of the mechanical and piezoelectric characteristics change during or after temperature variation.

\section{Conclusion}

In conclusion, we can notice through the results that the systematic increase in the temperature during the manufacture of smart composites with thermoplastic matrix causes an exceeding of the piezoelectric thermal limit. This fact makes a degrading in the ceramic crystal polarization of the piezoelectric components, i.e. the loss in the piezoelectric properties. 


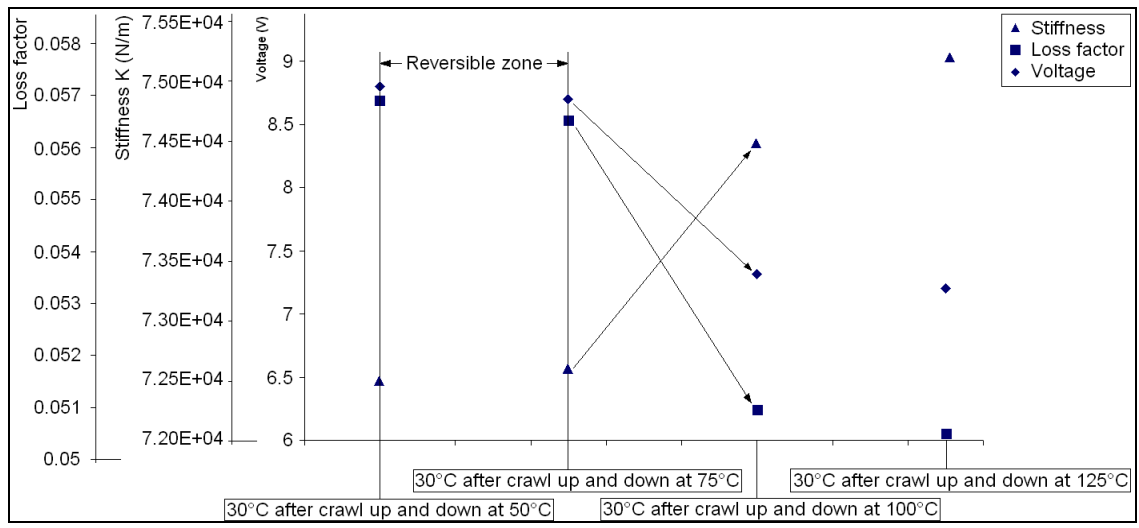

Figure 11: Variation curves of various parameters with the temperature increment measured by viscoanalyzer.

The empirical results obtained by viscoanalyzer show a beginning of this phenomenon from a temperature of approximately $75^{\circ} \mathrm{C}$ : until this temperature the voltage is reversible after each temperature crawl up and down. In addition, simulations show an important influence of the piezoelectric positioning component and the molded plate dimensions.

In spite of these facts which are compatible in certain cases and incompatible in others, a comparison was made between modeling and experiment. This comparison shows the requirement of integrating the piezoelectric component in a position which takes into consideration two effects: On one hand the liquid plastic filling effect (solidification of the plastic on surfaces and the temperature increment of piezoelectric component). On the other hand the thickness of the manufactured model. These results constitute a beneficial contribution for the smart thermoplastic composite manufacture.

\section{Acknowledgement}

The authors wish to acknowledge that this work was supported by the Francophone University Agency (AUF).

\section{References}

[1] Cracknell, Dyson, Handbook Of Thermoplastics Injection Mould Design, Blackie Academic \& Professional, pp. 10-80, 1993.

[2] Hori, Aoki, Ohira, Yano, New type of mechanical damping composites composed of piezoelectric ceramics, carbon black and epoxy resin. Composites Part A: Applied Science and Manufacturing, 32(2), pp. $287-$ 290, 2001.

[3] Tzou, Tseng, Distributed piezoelectric sensor/actuator design for dynamic measurement/control of distributed parameter systems: a piezoelectric finite element approach. J. Sound Vib., 138, pp. 17-34, 1990. 
[4] Agrawal B. N., Treanor K. E., Shape control of a beam using piezoelectric actuators. Smart Mater. Struct., 8, pp. 729-40, 1999.

[5] Brei, Moskalik, Deflection performance of a bi-directional distributed polymeric piezoelectric micromotor. J. Microelectromech. Syst., 6, pp. 62-9, 1997.

[6] Ervin, Brei, Recurve piezoelectric-strain-amplifying actuator architecture. IEEE/ASME Trans. Mechatron., 3, pp. 293-301, 1998.

[7] Eitel, Randall, Shrout, Rehrig, Hackenberger, Park, New High Temperature Morphotropic Phase Boundary Piezoelectrics Based on $\mathrm{Bi}(\mathrm{Me}) \mathrm{O}_{3}-\mathrm{PbTiO}_{3}$ Ceramics. The Japan Society of Applied Physics, 40(1), pp. 5999-6002, 2001.

[8] Mousseau, Sarda, Deterre, Thermique de l'injection des thermoplastiques /Optimisation. Technique de l'ingénieur, AM 3 685, pp. 1-18, 2005.

[9] MATWEB, www.matweb.com 